\title{
Autozygome-guided exome sequencing in retinal dystrophy patients reveals pathogenetic mutations and novel candidate disease genes
}

\author{
Leen Abu-Safieh, ${ }^{1,15}$ May Alrashed, ${ }^{1,2,3,15}$ Shamsa Anazi, ${ }^{1}$ Hisham Alkuraya, ${ }^{1,4}$ \\ Arif O. Khan, ${ }^{5}$ Mohammed Al-Owain, ${ }^{6,7}$ Jawahir Al-Zahrani, ${ }^{1}$ Lama Al-Abdi, ${ }^{1}$ \\ Mais Hashem, ${ }^{1}$ Salwa Al-Tarimi, ${ }^{1}$ Mohammed-Adeeb Sebai, ${ }^{1}$ Ahmed Shamia, ${ }^{1}$ \\ Mohamed D. Ray-zack, ${ }^{1}$ Malik Nassan, ${ }^{1}$ Zuhair N. Al-Hassnan, ${ }^{6,7}$ Zuhair Rahbeeni, ${ }^{6}$ \\ Saad Waheeb, ${ }^{8,9}$ Abdullah Alkharashi, ${ }^{10}$ Emad Abboud, ${ }^{11}$ Selwa A.F. Al-Hazzaa, ${ }^{1,12,13}$ \\ and Fowzan S. Alkuraya ${ }^{1,7,14,16}$
}

${ }^{1}$ Department of Genetics, King Faisal Specialist Hospital and Research Center, Riyadh 11211, Saudi Arabia; ${ }^{2}$ UCL Institute of Ophthalmology, Department of Genetics, London ECIV 9EL, United Kingdom; ${ }^{3}$ Department of Clinical Laboratory Sciences, College of Applied Medical Sciences, King Saud University, Riyadh 11573, Saudi Arabia; ${ }^{4}$ Department of Ophthalmology, College of Medicine, Imam Muhammed Bin Saud Islamic University, Riyadh 13317, Saudi Arabia; ${ }^{5}$ Department of Pediatrics, King Khaled Eye Specialist Hospital, Riyadh 11462, Saudi Arabia; ${ }^{6}$ Department of Medical Genetics, King Faisal Specialist Hospital and Research Center, Riyadh 11211, Saudi Arabia; ${ }^{7}$ Department of Anatomy and Cell Biology, College of Medicine, Alfaisal University, Riyadh 11533, Saudi Arabia; ${ }^{8}$ Department of Ophthalmology, King Faisal Specialist Hospital and Research Center, Jeddah 21499, Saudi Arabia; ${ }^{9}$ Department of Ophthalmology, College of Medicine, King Abdulaziz University, Jeddah 21352, Saudi Arabia; ${ }^{10}$ Department of Ophthalmology, College of Medicine, King Saud University, Riyadh 11573, Saudi Arabia; ${ }^{11}$ Department of Retina, King Khaled Eye Specialist Hospital, Riyadh 11462, Saudi Arabia; ${ }^{12}$ Department of Ophthalmology, King Faisal Specialist Hospital and Research Center, Riyadh 11211, Saudi Arabia; ${ }^{13}$ Department of Ophthalmology, College of Medicine, Alfaisal University, Riyadh 11533, Saudi Arabia; ${ }^{4}$ Department of Pediatrics, King Khalid University Hospital and College of Medicine, King Saud University, Riyadh 11573, Saudi Arabia

Retinal dystrophy (RD) is a heterogeneous group of hereditary diseases caused by loss of photoreceptor function and contributes significantly to the etiology of blindness globally but especially in the industrialized world. The extreme locus and allelic heterogeneity of these disorders poses a major diagnostic challenge and often impedes the ability to provide a molecular diagnosis that can inform counseling and gene-specific treatment strategies. In a large cohort of nearly 150 RD families, we used genomic approaches in the form of autozygome-guided mutation analysis and exome sequencing to identify the likely causative genetic lesion in the majority of cases. Additionally, our study revealed six novel candidate disease genes (C21orf2, EMC1, KIAA1549, GPR125, ACBD5, and DTHDI), two of which (ACBD5 and DTHDI) were observed in the context of syndromic forms of RD that are described for the first time.

[Supplemental material is available for this article.]

Deprivation of visual perception is a major form of morbidity worldwide with a wide array of causes that cover the entire spectrum from primarily environmental to primarily genetic. Representing the Mendelian end of the spectrum, retinal dystrophy (RD) is a vast group of blinding diseases that are characterized by loss of photoreceptor function, usually due to mono- or biallelic mutations in an expansive list of genes (Wright et al. 2010). Collectively, RD is a major cause of blindness, particularly in industrialized countries where infectious causes are less common and where treatable blinding diseases such as cataract and glaucoma receive adequate management.

Clinically, $\mathrm{RD}$ can take various forms, retinitis pigmentosa (RP) being the most common (Buch et al. 2004). RP patients typ-

\footnotetext{
${ }^{15}$ These authors contributed equally to this work.

${ }^{16}$ Corresponding author

E-mail falkuraya@kfshrc.edu.sa

Article published online before print. Article, supplemental material, and publication date are at http://www.genome.org/cgi/doi/10.1101/gr.144105.112.
}

ically present with a predominantly rod dysfunction, which manifests as night blindness, progressively worsening peripheral vision, and typical fundus appearance (Ho 2003; Hamel 2006). In cone dystrophies, it is the cone photoreceptors that are primarily involved, causing a substantial decrease in visual acuity and photophobia (Hamel 2007). In both classes, the other photoreceptor subtype is inevitably affected as the disease progresses, hence the terms rod-cone and cone-rod dystrophy, although the mechanism for this sympathetic cell loss is poorly understood. When severe $\mathrm{RD}$ is congenital or early-infantile in onset, it is usually referred to as Leber congenital amaurosis (LCA). Interestingly, the clinical boundaries between these subclasses are blurred by the increasing appreciation of the marked phenotypic variability that is associated with mutations in a large number of RD genes (Daiger et al. 2007).

The remarkable genetic heterogeneity (179 genes as of January 2012; https://sph.uth.tmc.edu/Retnet/sum-dis.htm) and the poor predictive value of the clinical assessment to the specific genetic etiology (at least in nonsyndromic cases) make it extremely chal- 
lenging to offer a molecular diagnosis to these patients (Koenekoop et al. 2007). Thus, of all Mendelian disorders, this is one disease category where most patients remain unaware of their underlying causative mutation even though such information is critical for informed genetic counseling that aims at prevention and expansion of available reproductive options. This is compounded by estimates that, even if all known RD were to be sequenced in a given patient, the yield is probably 50\% (Farrar et al. 2002; Hartong et al. 2006; den Hollander et al. 2008). An additional value in securing a molecular diagnosis lies in the recent progress in gene therapy, which has prompted many RD patients to seek to determine their mutation status in order to know whether they are eligible for these gene-specific treatment protocols (Maguire et al. 2008). In addition, certain classes of mutations have been found to be amenable to treatment in other diseases, e.g., nonsense mutations, which offers hope that RD patients with such mutations could similarly benefit from such innovative strategies, but this will require prior knowledge of the underlying genetic defect (Kerem et al. 2008).

Research in the genetics of RD has greatly improved our understanding of the molecular machinery that enables the retina to play a critical role in the perception of visual stimuli (Inglehearn 1998). While some of the genes were predicted to cause RD based on established physiological roles of the protein they encode, e.g., phototransduction genes, it came as a surprise that almost one in four RD genes plays a role in the photoreceptor cilium (Adams et al. 2007; Wright et al. 2010). Moreover, many genes were completely unsuspected, e.g., pre-mRNA splicing genes, and the function of some remains unknown (Vithana et al. 2001; Faustino and Cooper 2003; Wright et al. 2010). Indeed, the increasing pace of discovery of RD genes over the past few years has widened the gap between our knowledge of the genetic architecture of RD and its functional context.

In this study, we aimed to investigate the utility of genomic approaches in the study of RD genetics. Specifically, we implemented autozygome analysis (Woods et al. 2006; Alkuraya 2010) and exome sequencing in a large cohort of simplex and multiplex patients with different clinical RD subtypes. In addition to providing the most comprehensive analysis to date on the actual contribution of known RD genes to the overall mutation pool, our study reveals six novel RD genes, including two involved in novel syndromic forms of $\mathrm{RD}$, and suggests a framework for mutation identification in these patients.

\section{Results}

\section{Clinical characteristics}

For the period January 2008-September 2010, 149 eligible families were enrolled representing the three major clinical subtypes of RD, i.e., RP, LCA, and cone-rod dystrophy, but other less common phenotypes such as achromatopsia were also represented. With the exception of cone-dystrophy with supranormal rod response patients in whom $K C N V 2$ was directly sequenced, it was not possible to predict the genetic defect based on the phenotype provided, and they were processed as per the workflow outlined in

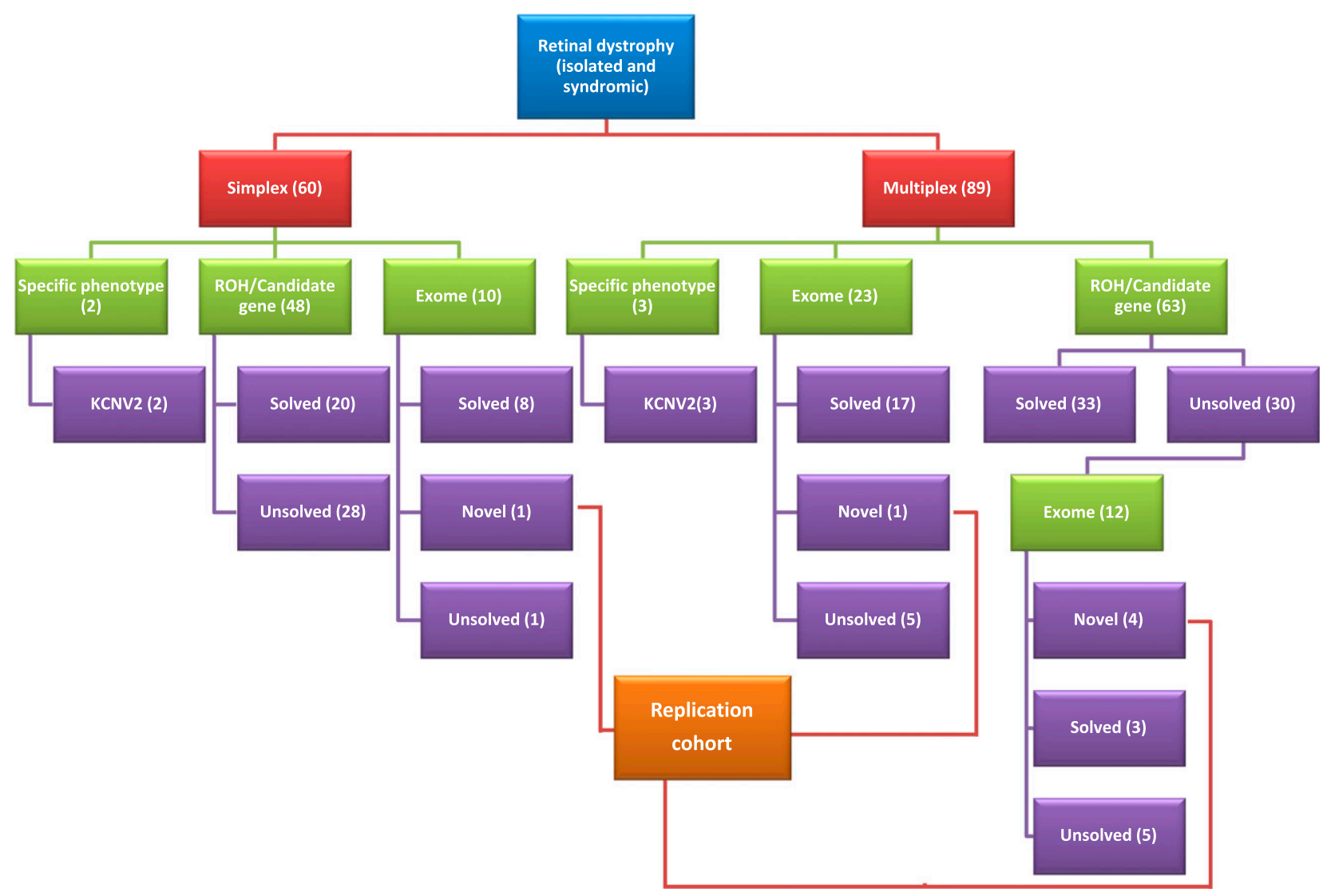

Figure 1. Workflow of the study. 
Figure 1. As expected, RP accounted for the majority of patients $(\sim 55 \%)$. The majority of cases were multiplex (60\%), and there was remarkable clinical homogeneity among affected members of a given family. Family arRP-FD02 (Supplemental Fig. S1) is worth highlighting in this regard. In this family of four affected members with RP (two siblings on either side of a first cousin relationship), two siblings on one side had additional features of Bardet-Bied syndrome (BBS). Although the two siblings in the other branch lacked any additional BBS feature, one of these two siblings had an infant with BBS in the course of the study. As a result, and based on our experience of rare cases in which BBS presents as nonsyndromic RP (Abu Safieh et al. 2010, 2012), we enrolled this family in this study. However, autozygome analysis and exome sequencing confirmed that the apparent clinical heterogeneity in this family was, in fact, the result of independent segregation of two different diseases: RP secondary to RP1 mutation and BBS due to BBS1 mutation. In Family arRP-F026, which was enrolled as nonsyndromic RP, the finding of a BBS4 mutation prompted us to recall the family for careful phenotyping, and the result indicated that the phenotype should have been labeled as BBS. On the other hand, Family CRF008, in which we identified a novel MKS1 mutation, was found upon rephenotyping to have no syndromic features. Thus, this appears to be the first report of MKS1 mutation causing nonsyndromic cone-rod dystrophy.

\section{Autozygome-guided gene sequencing}

This was pursued in both simplex and multiplex cases because of our past experience of the very high rate of homozygous mutations even in the absence of consanguinity or positive family history (Aldahmesh et al. 2009). The yield was only slightly lower in simplex compared with multiplex cases ( $42 \%$ vs. $52 \%$ ) (Fig. 2 ).

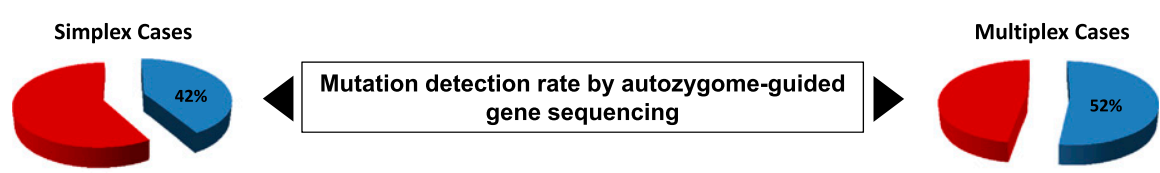

Genetic Architecture of RD in Mutation-positive Cases
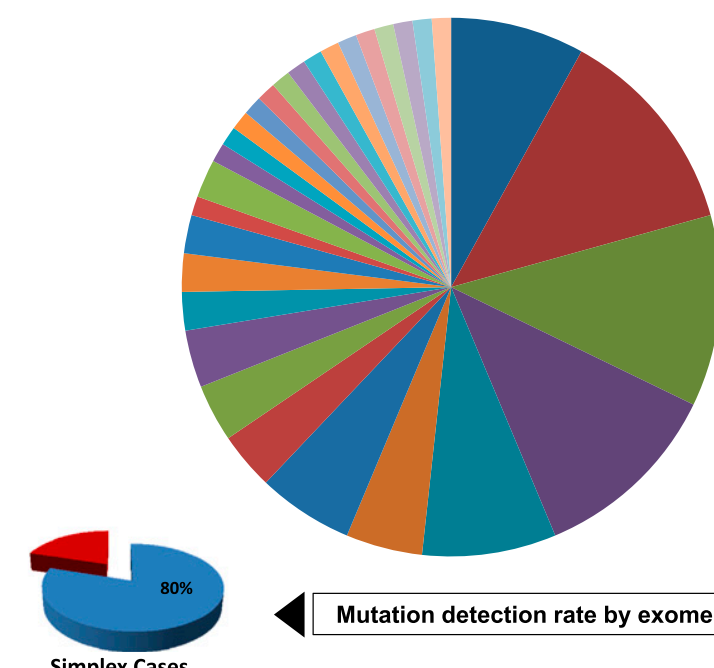

$\because K C N V 2 \quad \square R P 1$

TULP1 $\quad$ RPGRIP1

- CRB1 $\quad$ RPE65

$\square R D H 12 \quad \square$ EYS

NR2E3 $\quad A B C A 4$

$\square$ PRPH2 $\square L B P 1$

-CABP4 $\square$ MERTK

$\triangle$ CNGA3 $\square B P 3$

- C2orf71 $\square$ PROM1

$\triangle$ AIPL1 $B B S 1$

$\square B S 4 \quad \square C D H 21$

$\square$ PDE6B $\square$ PDEGA

$\square$ MKS1 $\quad$ CNGB1

$\triangle$ CERKL $\square$ IMPG2

CNNM4

Mutation detection rate by exome sequencing

Simplex Cases

Figure 2. Central pie chart summarizes the contribution of various genes to the overall mutational pool among RD patients in the current study. Pie charts in the upper panel show the percentage of mutation-positive cases among simplex and multiplex cases using the autozygome-guided gene sequencing approach. Pie charts in the lower panel show the percentage of mutation-positive cases among simplex and multiplex cases using the exome sequencing approach. Please note the percentages in these charts do not take into account the novel candidate genes identified in this study.
On average, four genes were sequenced per case (range 1-11). he average number of amplicons per case was 200, with an erage cost of $\$ 3000$. The results of autozygome-guided tarRD gene sequencing are summarized in Table 1 and Supemental Table S1 (solved cases) and Supplemental Table S4

\section{ome sequencing for mutation detection in RD} 23 multiplex cases to investigate the yield of this method in radic and familial cases of this extremely heterogeneous disathogenic mutations in known RD genes. A similar ratio (17/23, $4 \%$ ) was observed in multiplex cases (Fig. 2 ). In all these cases, the ental Table S2). By checking these mutations against the autogome data we had prepared for this purpose, it was clear that all 作 ne of the largest four runs of homozygosity $(\mathrm{ROH})$ (in simplex cases) (Supplemental Table S5) or an $\mathrm{ROH}$ that was exclusively ared by the affected members of a given family (in multiplex ces). However, there is a significant time and money difference verome sequencing. Indeed, a typical turnaround time ared to $15 \mathrm{wk}$ for autozygome-guided analysis. The cost of $\$ 1500$ ex exome was also lower than the $\$ 3000$ per case solved using the atozygome-guided approach. Furthermore, exome sequencing vealed mutations in two multiplex cases that were missed by the autozygome-guided approach because these mutations were homozygous regions which were shared with an unaffected individual, yet the apparently shared $\mathrm{ROH}$ was clearly IBS (identical-by-state) (Supplemental Fig. S2).

\section{Exome as a discovery tool for novel $\mathrm{RD}$ genes}

The first group of exomes (see above) also revealed two novel candidate genes for RD (Tables 3, 4). In Family sRP-001 (multiplex), a truncating mutation in $L G A L S 9 B$ encoding Galectin-9B protein A2 and a missense mutation in EMC1 were the only variants that survived filtration (Table 3; Supplemental Table S3; Supplemental Fig. S4). However, direct full sequencing of both genes in the 11 patients, out of 210 total RD patients in the replication cohort, whose autozygome overlapped with at least one of the two genes revealed one patient who is homozygous for the same mutation in EMC1 but no additional alleles of $L G A L S 9 B$. Importantly, the same LGALS9B truncation was later found at high frequency on direct sequencing of additional ethnically matched controls. On the other hand, the novel EMC1 variant was absent from 380 Saudi controls by direct sequencing and in the Exome Variant Server and is highly 


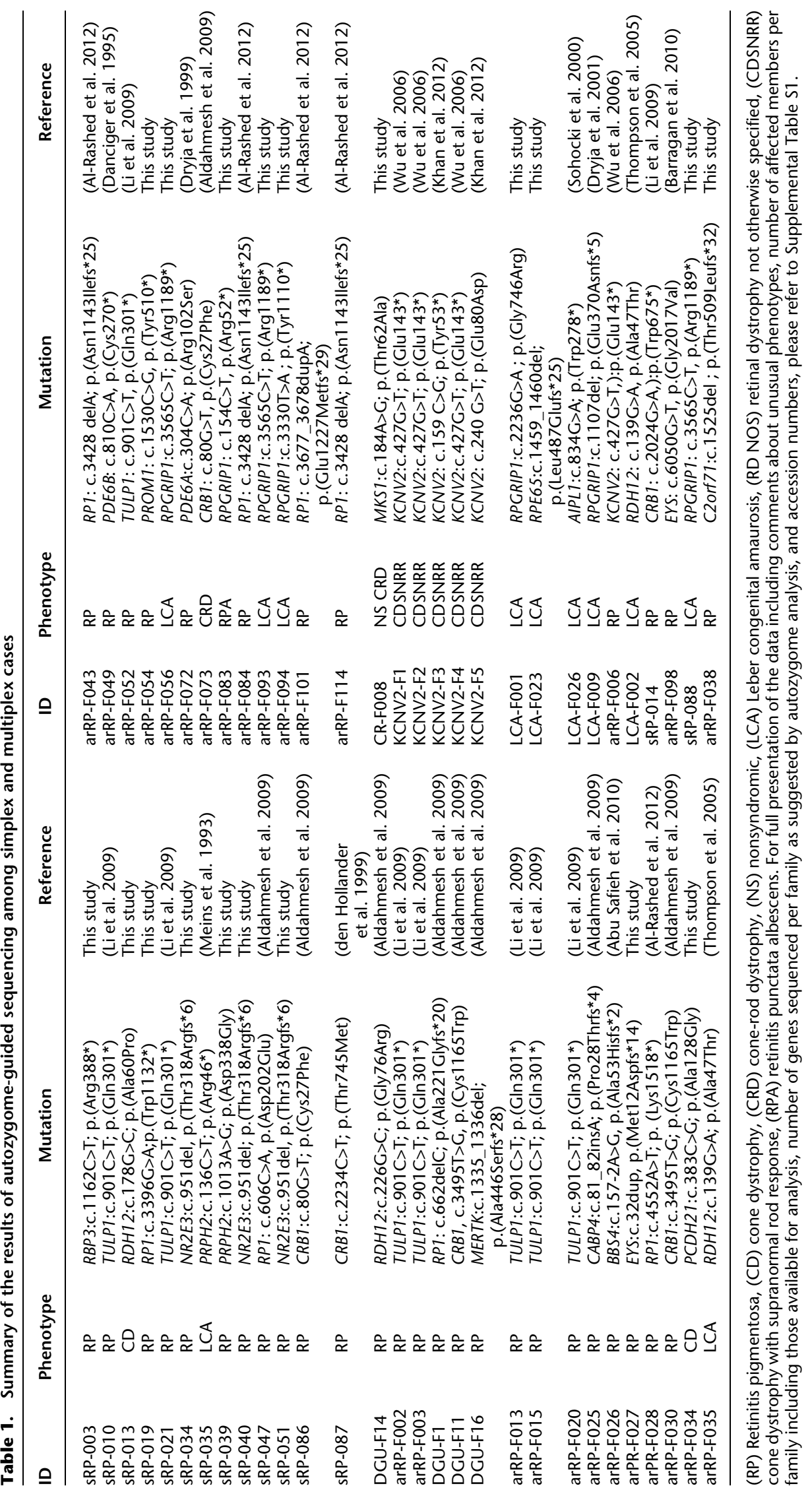




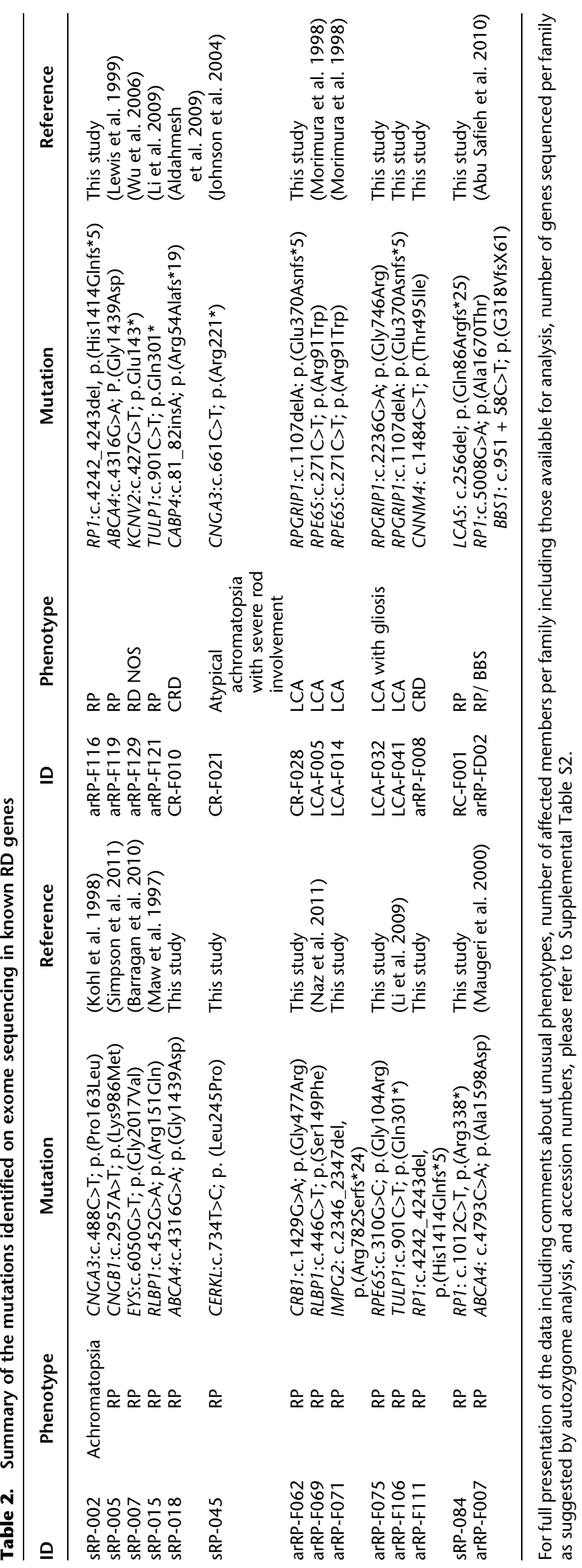




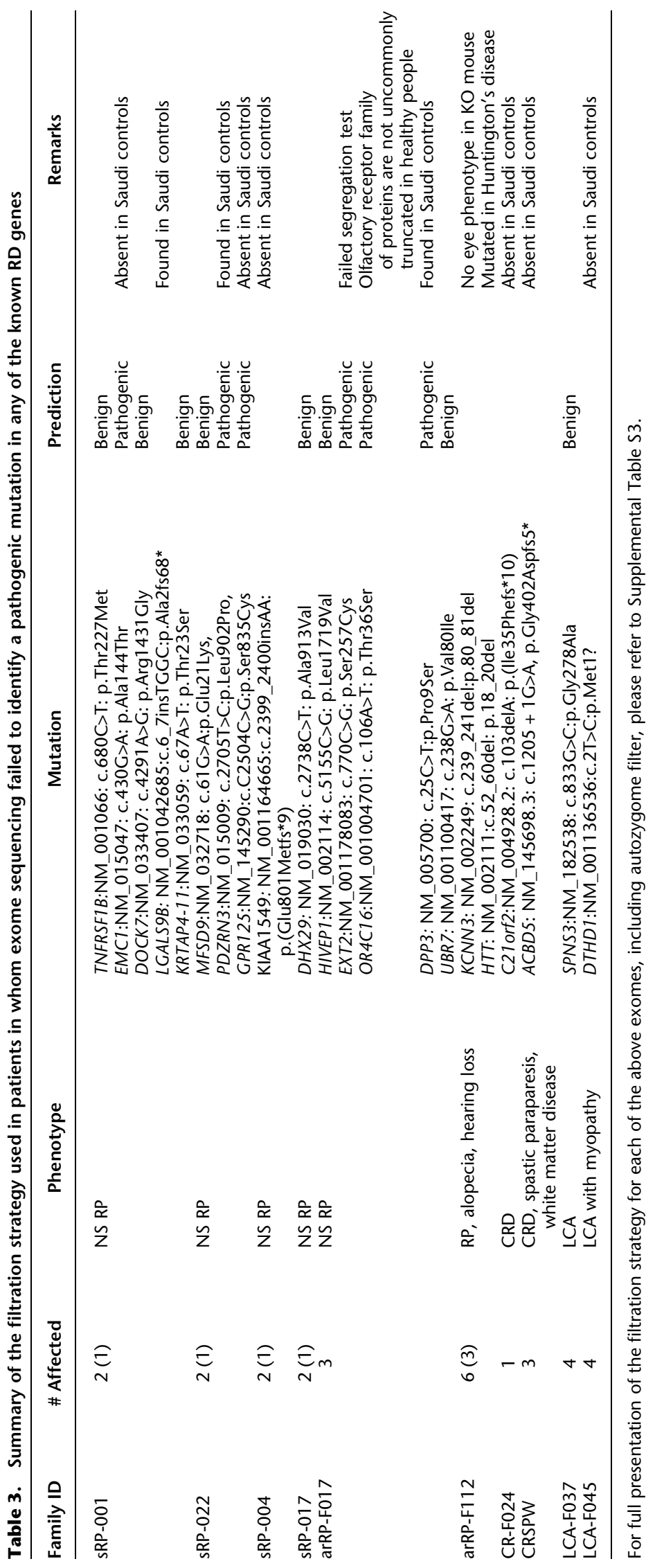




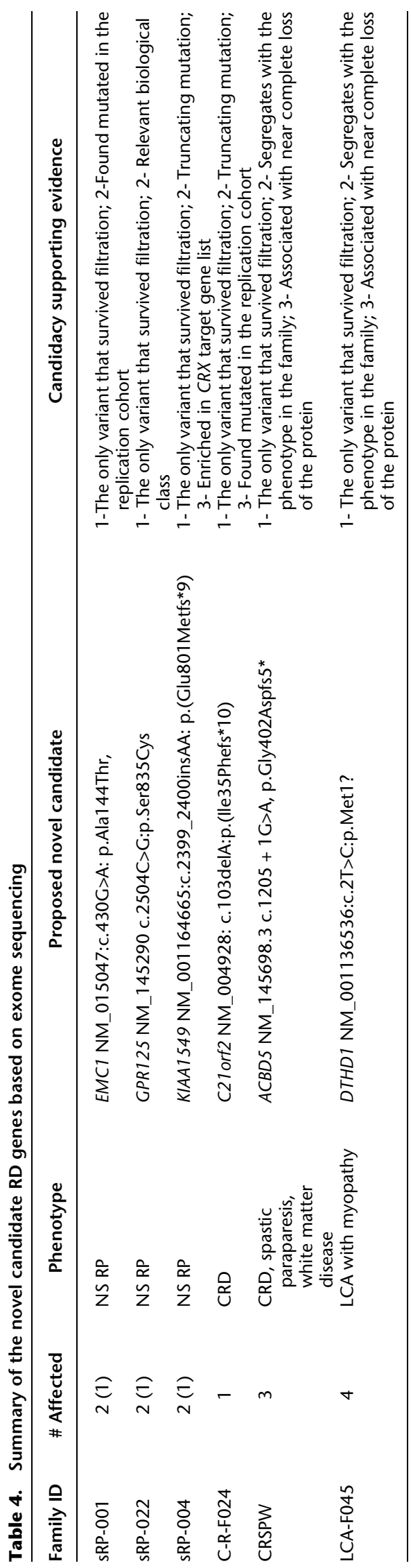


conserved across species. Taken together, these data strongly support the candidacy of $E M C 1$ in the pathogenicity of RP in the two individuals who are homozygous for that variant, although very little is known about this gene. In Family CR-F024 (simplex), a truncating mutation in the hypothetical protein coding gene C21orf2 was the only variant that survived the various filters. As with the EMC1, this was absent in 160 Saudis by exome sequencing and in 190 Saudi controls by direct sequencing and in the Exome Variant Server (EVS). More importantly, by direct sequencing in the seven individuals in the replication cohort whose autozygome pattern overlapped with this gene, one patient was found to be homozygous for a splicing mutation (NM_004928.2:c.545 + 1G>A) that fully abolishes the donor site in silico, which was absent in the panels of controls described above. Thus, C21orf2 is a compelling candidate in the pathogenesis of cone-rod dystrophy in these two individuals.

The second group of exomes $(n=12)$ was enriched for novel gene discovery because all known RD genes had been excluded in these multiplex families by the autozygome-guided sequencing approach. As mentioned above, despite this enrichment, 3/12 harbored mutations in known RD genes that were missed for various reasons (two because of IBS [identical by state] being confused with IBD [identical by descent], and one because of a highly unusual pedigree structure; see Supplemental Figs. S1, S2). A novel candidate gene was identified in each of four additional families (Tables 3, 4). In Family sRP-022 (multiplex), a novel missense mutation was identified in an absolutely conserved residue of the sixth transmembrane helix of $G$ protein-coupled receptor 125 (GPR125). Of note, mutations in several other G protein-coupled receptors are known to cause RD (Dryja et al. 1990; Morimura et al. 1999; Ebermann et al. 2009; Hilgert et al. 2009). In Family sRP-004 (multiplex), a novel truncating variant was identified in KIAA1549 as the only variant that remained after applying the various filters. Virtually nothing is known about this hypothetical protein-coding gene. However, it is among the top $4 \%$ of genes enriched for CRXbinding sites in a data set used to identify MAK as a novel RD gene (Ozgul et al. 2011; Tucker et al. 2011). Additionally, while the reduction of MAK representation in retina of mice with loss of photoreceptors was $\sim 26 \%$, that of KIAA1549 was $\sim 88 \%$, suggesting specific loss of this gene in photoreceptor degeneration (Ozgul et al. 2011). As with the GPR125 variant, this truncating variant was not encountered in any of 160 Saudi exomes or 190 Saudi controls by direct sequencing. Both were also absent in the EVS. These data support the candidacy of these two genes as novel RD genes. However, direct sequencing of both in the 27 patients in the replication cohort whose autozygome overlapped with either of these two genes revealed no additional mutations.

Two families displayed an apparently novel syndromic form of RD. In Family LCA-F045, LCA segregated with a mild-moderate form of nonspecific muscle dystrophy. By only considering the exome variants within the three exclusively shared $\mathrm{ROH}$ among the affected members, we uncovered a single nucleotide substitution that abolishes the first methionine residue of DTHD1 encoding death domain-containing protein 1 (Supplemental Fig. S3). Western blot analysis showed a greater than fourfold reduction in the abundance of the mutant protein compared to control (Fig. 3). Virtually nothing is known about this hypothetical protein other than that it contains a death domain. However, the identification of this as the only variant within the shared $\mathrm{ROH}$, its effect on the protein, its full segregation with the phenotype in this extended family, and its absence in a large number of controls strongly support its candidacy as the causal gene for this appar-
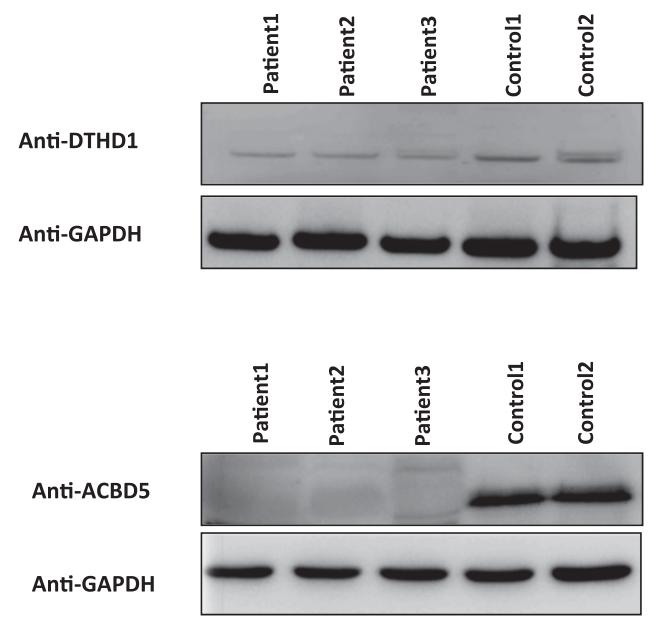

Figure 3. Western blot analysis of DTHD1 and ACBD5 in two families representing novel syndromic forms of RD. Fourfold reduction in the DTHD1 intensity in the patients compared to control and near-absence of the band corresponding to ACBD5 among patients can be seen. GAPDH is used for a loading control.

ently novel LCA/muscular dystrophy syndrome. In Family CRSPW, an apparently novel association between cone-rod dystrophy and psychomotor delay associated with significant white matter involvement was observed. A single novel variant was identified in the single ROH that is exclusively shared by the three affected siblings (Supplemental Fig. S3). The variant is predicted to abolish a consensus splice donor site in ACBD5. Indeed, RT-PCR confirmed the resulting aberrant transcript that predicts frameshift and premature truncation. However, despite lack of evidence of nonsense-mediated decay (NMD), Western blot analysis showed no evidence of the expected smaller band as a result of the truncation (the normal band was completely absent), even though the antibody targets the $\mathrm{N}$ terminus part of the protein (Fig. 3 ). Thus, it appears that the mutation causes severe instability of the protein and can be considered as a null allele. Reassuringly, as with the DTHD1 variant, this variant was absent in 160 Saudi exomes, 190 Saudi controls by direct sequencing, and EVS. ACBD5 encodes acyl-coenzyme A binding domain-containing protein 5, so it remains to be seen, as is the case with the above-mentioned novel candidates, how deficiency of this protein may have caused this phenotype.

In the remaining five families, no novel variants were identified after applying the various filters. Interestingly, linkage analysis in four of these families showed one single peak each (Chr17: 3,745,860-7,201,753 in LCA-F037, Chr3: 83,157,375107,875,119 in arRP-F048, Chr8: 75,000,000-110,000,000 in arRPF074, and Chr7:105,000,000-147,000,000 in arRP-F077) (Supplemental Table S6). In the remaining one family, we could not narrow the search to a single locus, so several ROHs were used in the filtration of the data.

\section{Discussion}

The extreme genetic heterogeneity of $\mathrm{RD}$ and the often poor predictive power of clinical assessment in determining the underlying genetic defect have severely hampered the ability of these patients to receive specific genetic diagnosis that can be the basis of informed genetic counseling and gene-specific therapy (Berger et al. 2010; den Hollander et al. 2010). Some attempts have been made 
to reduce this diagnostic challenge. In one approach, all previously reported mutations in $\mathrm{RD}$ genes were captured on a genotyping chip (Koenekoop et al. 2007). Unfortunately, the extreme allelic heterogeneity limits the usefulness of this method. The resequencing chip theoretically circumvents this limitation, but the prerequisite step of amplifying all known RD genes represents a major challenge (Booij et al. 2011). We and others have shown that the autozygome approach can be very effective in guiding the mutation analysis (Aldahmesh et al. 2009; Pomares et al. 2010). Interestingly, this approach was also used successfully in populations where consanguinity is uncommon (Hildebrandt et al. 2009; Collin et al. 2011; Hagiwara et al. 2011; Schuurs-Hoeijmakers et al. 2011). However, this approach has its limitations. Only homoallelic mutations are identified by this method, so compound heterozygosity for recessive RD genes, heterozygosity for dominant RD genes, and hemizygosity for X-linked RD genes are missed. More importantly, novel genes can only be identified in favorable pedigrees, i.e., those in which enough crossing-overs reduce the haplotype sharing to a level that allows a relatively small $\mathrm{ROH}$ to be identified that is exclusively shared by the affected members. Indeed, lining up the autozygome pattern of unrelated individuals, which has been used to identify disease loci for autosomal recessive traits in the past as a way to circumvent the limited informativeness of any given family, is largely inapplicable, given the remarkable locus heterogeneity of RD. Finally, as we show in this study, the distinction of IBS and IBD can be challenging (Alkuraya 2012).

Next-generation sequencing allows massively parallel sequencing at an unprecedented scale both in throughput and cost and has recently been used on a smaller scale in the study of retinal dystrophy genetics (Audo et al. 2012; Neveling et al. 2012). Exome sequencing is one of its applications where the protein-coding exons of all known genes can be captured, followed by highthroughput sequencing. Although deep intronic and noncoding regulatory sequence mutations are not covered by this method, we hypothesized that it still lends itself as a powerful genomic tool to at once identify mutations in known RD genes and identify novel $\mathrm{RD}$ genes, and we set out to investigate its utility both in isolation and in combination with the autozygome approach.

Our data show autozygome-guided sequence analysis of known RD genes is applicable to both multiplex and simplex cases, which suggests that, even in simplex cases, autosomal recessive RD is the commonest form, at least in our population that is characterized by a high rate of consanguinity. Although a few founder mutations were identified, we find that, similar to our experience with other genetically heterogeneous conditions, there is marked allelic and locus heterogeneity in our population, even within the same tribe. However, we caution against the overinterpretation of this phenomenon as being indicative of high population genetic diversity akin to what is observed in Africa, without empirical population genetic data, which still do not exist for Arabia.

An important yet largely unanswered question is how much the current list of RD genes contributes to the overall genetic architecture of this disease. Only estimates are available because, until recently, the only way to empirically test this was through the PCR amplification of all RD genes, an extremely challenging task. By performing exome sequencing on randomly selected multiplex and simplex cases, we were able to show that the genes identified as of January 2012 account for $74 \%-80 \%$ of the overall mutation pool in our population. Interestingly, all mutations identified by exome sequencing of simplex cases were homoallelic even though hemizygous X-linked, and compound heterozygous mutations in all known RD genes were equally likely to be identified. Indeed, the comparable yield of unselected exomes in simplex and multiplex cases argues against a major contribution of X-linked RD genes in simplex cases in our population. It is unclear how applicable this result is to more outbred populations, although evidence suggests that many sporadic patients in those populations also represent autosomal recessive inheritance (Avila-Fernandez et al. 2010; Iwanami et al. 2012). Another important result from our study is that our exome sequencing data make it unlikely that any additional novel gene will account for a substantial fraction of the remaining cases (see below).

As predicted, in addition to revealing most mutations in known $\mathrm{RD}$ genes, exome sequencing was a useful discovery tool as well. We and others have previously demonstrated the power of exome sequencing in revealing novel disease genes based on simplex cases (Gilissen et al. 2010; Aldahmesh et al. 2011; Shaheen et al. 2011). Our data expand the disease phenotypes for which simplex cases can be used to identify novel disease genes to also include RD. Unfortunately, the very low contribution of most RD genes to the overall mutation pool makes it challenging to identify additional pathogenic alleles in the candidate genes we identified in this study, so they remain interesting candidates pending independent verification by future studies (at least in the four for which no additional mutation-positive patients were identified in the replication cohort).

Many syndromes are known to involve the RD phenotype (Ayuso et al. 1995). However, we are not aware of any previously described association between LCA and muscular dystrophy or between cone-dystrophy and severe white matter disease. Thus, we believe these are two novel syndromic forms of RD. In both families, compelling loss of function alleles were identified (DTHD1 and $A C B D 5$ ), but additional work is needed to explore the presumed causal link mechanistically. These families were part of a collection we tried to enrich for novel RD genes. However, we show how pitfalls in homozygosity scan caused false negative results in three of the 11 families. In fact, the $13.3-\mathrm{Mb}$ IBS that caused confusion in the analysis of Family arRP-F069 is the largest IBS that we are aware of (Supplemental Fig. S2; Alkuraya 2012). Thus, it is possible that the higher yield of exome compared to autozygomeguided analysis can be, at least in part, caused by occasional pitfalls in homozygosity scan. Overall, we show that exome sequencing was superior to the autozygome-guided approach, and although the latter can be very helpful in lending credence to novel disease genes, it does not appear necessary in interpreting exome variants in known RD genes.

In summary, in this largest comprehensive genomic study of RD patients to date, we show that genomic tools are very useful in identifying the underlying genetic lesion. Exome sequencing in particular appears to be an attractive first-line test without prior enrichment for known RD genes, especially with its constantly decreasing cost. The novel disease genes we identified require validation in independent patient cohorts. Similar studies on outbred populations will be needed to explore potential differences in the genetic architecture of RD compared to what we presented in this study.

\section{Methods}

\section{Human subjects}

Patients with RP, LCA, and cone-rod dystrophy were actively recruited regardless of their age or family history through a wide network of ophthalmologists that covers all regions of Saudi Arabia for the period January 2008 to September 2010. Patients recruited

\section{Genome Research}


between September 2010 and May 2012 were only used as a "replication cohort" for the purpose of identifying additional mutations in the novel candidate genes we may identify in the main cohort. Assignment to a specific clinical subtype was based on clinical and, in selected cases, electrophysiological assessment. Syndromic patients were only considered further if they did not fit the clinical description of a known syndrome, e.g., Usher, BardetBiedl, Alstrom, and Joubert syndrome (these patients were recruited for other projects). Pedigrees were drawn for all recruited patients, and an effort was made to enroll additional affected relatives when present. Whenever possible, we enrolled parents and unaffected siblings for segregation analysis. All subjects signed an IRB-approved written informed consent (RAC\# 2070 023), followed by venous blood sampling in EDTA tubes. For selected patients, we also obtained blood samples in sodium heparin tubes followed by establishment of EBV-transformed lymphoblast cell lines for RNA and protein studies.

\section{Workflow}

Figure 1 summarizes the algorithm we implemented in the study which is described below in detail.

\section{Autozygome analysis}

Genotyping was performed on an Affymetrix Axiom or Affymetrix 250K SNP chip platform following the manufacturer's instructions on the index only (in simplex cases) and the entire sibship, when possible (in multiplex cases). Autozygome analysis was performed using Genotyping Console (Affymetrix) or autoSNPa as described before (Carr et al. 2006). In simplex cases, we only considered the four largest runs of homozygosity $(\mathrm{ROH})$ initially, but if negative, we expanded our search to all ROHs that are $>2 \mathrm{Mb}$ in size. In multiplex cases, we considered all ROHs that are exclusively shared by the affected members of a given sibship. All RD genes within an ROH were sequenced even when they appeared incompatible with the specific phenotype or pattern of inheritance to account for the known phenotypic variability of mutations in RD genes and the dual inheritance pattern for some of them. Twelve out of $30 \mathrm{mul}-$ tiplex cases in which autozygome-guided targeted RD gene sequencing failed to identify the causative mutation were processed for exome sequencing.

\section{Exome sequencing and analysis}

Two groups of samples were processed for exome sequencing. The first group represents randomly selected simplex (10) and multiplex (23) cases. The second group represents samples in which autozygome-guided targeted RD gene sequencing failed to identify the causative mutation by the first freeze point (12 out of 30) (Fig. 1). The aim of the first group was to investigate the utility of exome sequencing as a first-pass diagnostic test in $\mathrm{RD}$, whereas the aim was to enrich for novel RD genes in the second group. Exome capture was performed using the TruSeq Exome Enrichment kit (Illumina) following the manufacturer's protocol. Samples were prepared as an Illumina sequencing library, and in the second step, the sequencing libraries were enriched for the desired target using the Illumina Exome Enrichment protocol. The captured libraries were sequenced using an Illumina HiSeq2000 Sequencer. The reads were mapped against UCSC hg19 (http://genome.ucsc.edu/) by BWA (http://bio-bwa.sourceforge.net/). The SNPs and indels were detected by SAMtools (http://samtools.sourceforge.net/). For subsequent analysis, we always started by checking all genes reported to cause RD until January 2012. We considered homozygous, heterozygous, hemizygous, and compound heterozygous changes in these genes that are likely to be pathogenic, i.e., coding (excluding synonymous unless they affect splice site) or splice-site variants that are not present in 160 in-house Saudi exomes. It is important to mention that we manually checked all dbSNP variants in these genes against the Human Genome Mutation Database since a lot of previously reported pathogenic mutations are listed in dbSNP. Only when no such changes are identified did we proceed with the analysis of sequence variants following the filtration scheme outlined in Table 3 and Supplemental Figure S4. The autozygome filter refers to variants present within the four largest blocks of homozygosity in simplex cases and all blocks of shared homozygosity in multiplex cases.

\section{Replication analysis of novel candidate genes}

Novel candidate genes were fully sequenced in the "replication cohort" in search of additional alleles using standard PCR and Sanger sequencing. We specifically sequenced patients whose autozygome overlapped with any of these novel candidates.

\section{RT-PCR and immunoblotting}

Splice-site mutations were checked for potential effect on splicing in silico. Whenever possible, mutations that are likely to affect splicing were verified on RT-PCR using custom-made primers and lymphoblast-derived RNA as a template. Truncating mutations in novel genes were verified whenever possible by Western blot analysis using commercially available antibodies and lymphoblast-derived protein as the target and following standard protocols.

\section{Data access}

All novel sequence variants in known RD genes as well as those in the novel candidate genes that we report in this study have been submitted to the Leiden Open Variation Database (http://grenada. lumc.nl/LOVD2/eye/variants.php) under the following IDs: ABCA4_00014, ACBD5_00001, C21orf2_00001, C2orf71_00006, CDHR1_00001, CERKL_00001, CNNM4_00001, CRB1_00038, CRB1_00037, DTHD1_00001, EMC1_00001, EYS_00006, GPR125_ 00001, KIAA1549_00001, LCA5_00001, PRPH2_00003, RDH12_ 00002 .

\section{Acknowledgments}

We thank the patients and their families for their enthusiastic participation. We thank the Genotyping and Sequencing Core Facilities at KFSHRC for their technical help. This work was supported by KACST Grant 08MED497-20 (F.S.A.), DHFMR Collaborative Research Grant (F.S.A.), and PSCDR Research Grant (F.S.A.).

\section{References}

Abu Safieh L, Aldahmesh MA, Shamseldin H, Hashem M, Shaheen R, Alkuraya H, Al Hazzaa SA, Al-Rajhi A, Alkuraya FS. 2010. Clinical and molecular characterisation of Bardet-Biedl syndrome in consanguineous populations: The power of homozygosity mapping. J Med Genet 47: 236241.

Abu-Safieh L, Al-Anazi S, Al-Abdi L, Hashem M, Alkuraya H, Alamr M, Sirelkhatim MO, Al-Hassnan Z, Alkuraya B, Mohamed JY, et al. 2012. In search of triallelism in Bardet-Biedl syndrome. Eur J Hum Genet 20: 420 427.

Adams NA, Awadein A, Toma HS. 2007. The retinal ciliopathies. Ophthalmic Genet 28: 113-125.

Al-Rashed M, Abu Safieh L, Alkuraya H, Aldahmesh MA, Alzahrani J, Diya M, Hashem M, Hardcastle AJ, Al-Hazzaa SA, Alkuraya FS. 2012. RP1 and retinitis pigmentosa: Report of novel mutations and insight into mutational mechanism. British J Ophthalmol 96: 1018-1022. 
Aldahmesh MA, Safieh LA, Alkuraya H, Al-Rajhi A, Shamseldin H, Hashem M, Alzahrani F, Khan AO, Alqahtani F, Rahbeeni Z, et al. 2009. Molecular characterization of retinitis pigmentosa in Saudi Arabia. Mol Vis 15: 2464-2469.

Aldahmesh MA, Mohamed JY, Alkuraya HS, Verma IC, Puri RD, Alaiya AA Rizzo WB, Alkuraya FS. 2011. Recessive mutations in ELOVL4 cause ichthyosis, intellectual disability, and spastic quadriplegia. Am J Hum Genet 89: 745-750.

Alkuraya FS. 2010. Autozygome decoded. Genet Med 12: 765-771.

Alkuraya FS. 2012. Discovery of rare homozygous mutations from studies of consanguineous pedigrees. Curr Protoc Hum Genet 75: 6.12.1-6.12.13.

Audo I, Bujakowska KM, Leveillard T, Mohand-Said S, Lancelot ME, Germain A, Antonio A, Michiels C, Saraiva JP, Letexier M, et al. 2012. Development and application of a next-generation-sequencing (NGS) approach to detect known and novel gene defects underlying retinal diseases. Orphanet J Rare Dis 7: 8. doi: 10.1186/1750-1172-7-8.

Avila-Fernandez A, Cantalapiedra D, Aller E, Vallespin E, Aguirre-Lamban J, Blanco-Kelly F, Corton M, Riveiro-Alvarez R, Allikmets R, Trujillo-Tiebas MJ, et al. 2010. Mutation analysis of 272 Spanish families affected by autosomal recessive retinitis pigmentosa using a genotyping microarray. Mol Vis 16: 2550-2558.

Ayuso C, Garcia-Sandoval B, Najera C, Valverde D, Carballo M, Antinolo G. 1995. Retinitis pigmentosa in Spain. The Spanish multicentric and multidisciplinary group for research into retinitis pigmentosa. Clin Genet 48: 120-122.

Barragan I, Borrego S, Pieras JI, Gonzalez-del Pozo M, Santoyo J, Ayuso C, Baiget M, Millan JM, Mena M, Abd El-Aziz MM, et al. 2010. Mutation spectrum of EYS in Spanish patients with autosomal recessive retinitis pigmentosa. Hum Mutat 31: E1772-E1800.

Berger W, Kloeckener-Gruissem B, Neidhardt J. 2010. The molecular basis of human retinal and vitreoretinal diseases. Prog Retin Eye Res 29: 335-375.

Booij JC, Bakker A, Kulumbetova J, Moutaoukil Y, Smeets B, Verheij J, Kroes HY, Klaver CC, van Schooneveld M, Bergen AA et al. 2011. Simultaneous mutation detection in 90 retinal disease genes in multiple patients using a custom-designed 300-kb retinal resequencing chip. Ophthalmology 118: $160-167$.

Buch H, Vinding T, La Cour M, Appleyard M, Jensen GB, Nielsen NV. 2004 Prevalence and causes of visual impairment and blindness among 9980 Scandinavian adults: The Copenhagen City Eye Study. Ophthalmology 111: $53-61$.

Carr IM, Flintoff KJ, Taylor GR, Markham AF, Bonthron DT. 2006. Interactive visual analysis of SNP data for rapid autozygosity mapping in consanguineous families. Hum Mutat 27: 1041-1046.

Collin RW, van den Born LI, Klevering BJ, de Castro-Miro M, Littink KW Arimadyo K, Azam M, Yazar V, Zonneveld MN, Paun CC, et al. 2011. High-resolution homozygosity mapping is a powerful tool to detect novel mutations causative of autosomal recessive RP in the Dutch population. Invest Ophthalmol Vis Sci 52: 2227-2239.

Daiger SP, Bowne SJ, Sullivan LS. 2007. Perspective on genes and mutations causing retinitis pigmentosa. Arch Ophthalmol 125: 151-158.

Danciger M, Blaney J, Gao YQ, Zhao DY, Heckenlively JR, Jacobson SG, Farber DB. 1995. Mutations in the PDE6B gene in autosomal recessive retinitis pigmentosa. Genomics 30: $1-7$.

den Hollander AI, ten Brink JB, de Kok YJ, van Soest S, van den Born LI, van Driel MA, van de Pol DJ, Payne AM, Bhattacharya SS, Kellner U, et al. 1999. Mutations in a human homologue of Drosophila crumbs cause retinitis pigmentosa (RP12). Nat Genet 23: 217-221.

den Hollander AI, Roepman R, Koenekoop RK, Cremers FP. 2008. Leber congenital amaurosis: Genes, proteins and disease mechanisms. Prog Retin Eye Res 27: 391-419.

den Hollander AI, Black A, Bennett J, Cremers FP. 2010. Lighting a candle in the dark: Advances in genetics and gene therapy of recessive retinal dystrophies. J Clin Invest 120: 3042-3053.

Dryja TP, McGee TL, Reichel E, Hahn LB, Cowley GS, Yandell DW, Sandberg MA, Berson EL. 1990. A point mutation of the rhodopsin gene in one form of retinitis pigmentosa. Nature 343: 364-366.

Dryja TP, Rucinski DE, Chen SH, Berson EL. 1999. Frequency of mutations in the gene encoding the alpha subunit of rod cGMP-phosphodiesterase in autosomal recessive retinitis pigmentosa. Invest Ophthalmol Vis Sci 40: 1859-1865.

Dryja TP, Adams SM, Grimsby JL, McGee TL, Hong DH, Li T, Andreasson S, Berson EL. 2001. Null RPGRIP1 alleles in patients with Leber congenital amaurosis. Am J Hum Genet 68: 1295-1298.

Ebermann I, Wiesen MH, Zrenner E, Lopez I, Pigeon R, Kohl S, Lowenheim H, Koenekoop RK, Bolz HJ. 2009. GPR98 mutations cause Usher syndrome type 2 in males. J Med Genet 46: 277-280.

Farrar GJ, Kenna PF, Humphries P. 2002. On the genetics of retinitis pigmentosa and on mutation-independent approaches to therapeutic intervention. $E M B O$ J 21: 857-864.

Faustino NA, Cooper TA. 2003. Pre-mRNA splicing and human disease. Genes Dev 17: 419-437.
Gilissen C, Arts HH, Hoischen A, Spruijt L, Mans DA, Arts P, van Lier B, Steehouwer M, van Reeuwijk J, Kant SG, et al. 2010. Exome sequencing identifies WDR35 variants involved in Sensenbrenner syndrome. Am J Hum Genet 87: 418-423.

Hagiwara K, Morino H, Shiihara J, Tanaka T, Miyazawa H, Suzuki T, Kohda M, Okazaki Y, Seyama K, Kawakami H. 2011. Homozygosity mapping on homozygosity haplotype analysis to detect recessive disease-causing genes from a small number of unrelated, outbred patients. PLOS ONE 6: e25059. doi: 10.1371/journal.pone.0025059.

Hamel C. 2006. Retinitis pigmentosa. Orphanet J Rare Dis 1: 40. doi: 10.1186/ 1750-1172-1-40.

Hamel CP. 2007. Cone rod dystrophies. Orphanet J Rare Dis 2: 7. doi 10.1186/1750-1172-2-7.

Hartong DT, Berson EL, Dryja TP. 2006. Retinitis pigmentosa. Lancet 368: 1795-1809.

Hildebrandt F, Heeringa SF, Ruschendorf F, Attanasio M, Nurnberg G, Becker C, Seelow D, Huebner N, Chernin G, Vlangos CN, et al. 2009. A systematic approach to mapping recessive disease genes in individuals from outbred populations. PLoS Genet 5: e1000353. doi: 10.1371/ journal.pgen.1000353.

Hilgert N, Kahrizi K, Dieltjens N, Bazazzadegan N, Najmabadi H, Smith RJ, Van Camp G. 2009. A large deletion in GPR98 causes type IIC Usher syndrome in male and female members of an Iranian family. J Med Genet 46: $272-276$.

Ho AC. 2003. Retina: Color atlas and synopsis of clinical ophthalmology. McGraw-Hill, New York.

Inglehearn CF. 1998. Molecular genetics of human retinal dystrophies. Eye (Lond) 12: 571-579.

Iwanami M, Oshikawa M, Nishida T, Nakadomari S, Kato S. 2012. High prevalence of mutations in the EYS gene in Japanese patients with autosomal recessive retinitis pigmentosa. Invest Ophthalmol Vis Sci 53: 1033-1040.

Johnson S, Michaelides M, Aligianis IA, Ainsworth JR, Mollon JD, Maher ER, Moore AT, Hunt DM. 2004. Achromatopsia caused by novel mutations in both CNGA3 and CNGB3. J Med Genet 41: e20. doi: 10.1136/jmg. 2003.011437.

Kerem E, Hirawat S, Armoni S, Yaakov Y, Shoseyov D, Cohen M, NissimRafinia M, Blau H, Rivlin J, Aviram M, et al. 2008. Effectiveness of PTC124 treatment of cystic fibrosis caused by nonsense mutations: A prospective phase II trial. Lancet 372: 719-727.

Khan AO, Alrashed M, Alkuraya FS. 2012. 'Cone dystrophy with supranormal rod response' in children. Brit J Ophthalmol 96: 422-426.

Koenekoop RK, Lopez I, den Hollander AI, Allikmets R, Cremers FP. 2007. Genetic testing for retinal dystrophies and dysfunctions: Benefits, dilemmas and solutions. Clin Experiment Ophthalmol 35: 473-485.

Kohl S, Marx T, Giddings I, Jagle H, Jacobson SG, Apfelstedt-Sylla E, Zrenner E, Sharpe LT, Wissinger B. 1998. Total colourblindness is caused by mutations in the gene encoding the alpha-subunit of the cone photoreceptor CGMP-gated cation channel. Nat Genet 19: 257-259.

Lewis RA, Shroyer NF, Singh N, Allikmets R, Hutchinson A, Li Y, Lupski JR, Leppert M, Dean M. 1999. Genotype/phenotype analysis of a photoreceptor-specific ATP-binding cassette transporter gene, ABCR, in Stargardt disease. Am J Hum Genet 64: 422-434.

Li Y, Wang H, Peng J, Gibbs RA, Lewis RA, Lupski JR, Mardon G, Chen R. 2009. Mutation survey of known LCA genes and loci in the Saudi Arabian population. Invest Ophthalmol Vis Sci 50: 1336-1343.

Maguire AM, Simonelli F, Pierce EA, Pugh EN Jr, Mingozzi F, Bennicelli J, Banfi S, Marshall KA, Testa F, Surace EM, et al. 2008. Safety and efficacy of gene transfer for Leber's congenital amaurosis. N Engl J Med 358: $2240-$ 2248 .

Maugeri A, Klevering BJ, Rohrschneider K, Blankenagel A, Brunner HG, Deutman AF, Hoyng CB, Cremers FP. 2000. Mutations in the ABCA4 (ABCR) gene are the major cause of autosomal recessive cone-rod dystrophy. Am I Hum Genet 67: 960-966.

Maw MA, Kennedy B, Knight A, Bridges R, Roth KE, Mani EJ, Mukkadan JK, Nancarrow D, Crabb JW, Denton MJ. 1997. Mutation of the gene encoding cellular retinaldehyde-binding protein in autosomal recessive retinitis pigmentosa. Nat Genet 17: 198-200.

Meins M, Grüning G, Blankenagel A, Krastel H, Reck B, Fuchs S, Schwinger E, Gal A. 1993. Heterozygous 'null allele' mutation in the human peripherin/RDS gene. Hum Mol Genet 2: 2181-2182.

Morimura H, Saindelle-Ribeaudeau F, Berson EL, Dryja TP. 1999. Mutations in RGR, encoding a light-sensitive opsin homologue, in patients with retinitis pigmentosa. Nat Genet 23: 393-394.

Naz S, Ali S, Riazuddin SA, Farooq T, Butt NH, Zafar AU, Khan SN, Husnain T, Macdonald IM, Sieving PA, et al. 2011. Mutations in RLBP1 associated with fundus albipunctatus in consanguineous Pakistani families. Brit J Ophthalmol 95: 1019-1024.

Neveling K, Collin RW, Gilissen C, van Huet RA, Visser L, Kwint MP, Gijsen SJ, Zonneveld MN, Wieskamp N, de Ligt J, et al. 2012. Next-generation genetic testing for retinitis pigmentosa. Hum Mutat 33: 963-972.

\section{Genome Research}


Ozgul RK, Siemiatkowska AM, Yucel D, Myers CA, Collin RW, Zonneveld MN, Beryozkin A, Banin E, Hoyng CB, van den Born LI, et al. 2011. Exome sequencing and cis-regulatory mapping identify mutations in MAK, a gene encoding a regulator of ciliary length, as a cause of retinitis pigmentosa. Am J Hum Genet 89: 253-264.

Pomares E, Riera M, Permanyer J, Mendez P, Castro-Navarro J, AndresGutierrez A, Marfany G, Gonzalez-Duarte R. 2010. Comprehensive SNPchip for retinitis pigmentosa-Leber congenital amaurosis diagnosis: New mutations and detection of mutational founder effects. Eur J Hum Genet 18: $118-124$.

Schuurs-Hoeijmakers JH, Hehir-Kwa JY, Pfundt R, van Bon BW, de Leeuw N, Kleefstra T, Willemsen MA, van Kessel AG, Brunner HG, Veltman JA, et al. 2011. Homozygosity mapping in outbred families with mental retardation. Eur J Hum Genet 19: 597-601.

Shaheen R, Faqeih E, Sunker A, Morsy H, Al-Sheddi T, Shamseldin HE, Adly N, Hashem M, Alkuraya FS. 2011. Recessive mutations in DOCK6, encoding the guanidine nucleotide exchange factor DOCK6, lead to abnormal actin cytoskeleton organization and Adams-Oliver syndrome. Am J Hum Genet 89: 328-333.

Simpson DA, Clark GR, Alexander S, Silvestri G, Willoughby CE. 2011. Molecular diagnosis for heterogeneous genetic diseases with targeted high-throughput DNA sequencing applied to retinitis pigmentosa. J Med Genet 48: $145-151$.

Sohocki MM, Perrault I, Leroy BP, Payne AM, Dharmaraj S, Bhattacharya SS, Kaplan J, Maumenee IH, Koenekoop R, Meire FM, et al. 2000. Prevalence of AIPL1 mutations in inherited retinal degenerative disease. Mol Genet Metab 70: $142-150$.

Thompson DA, Janecke AR, Lange J, Feathers KL, Hubner CA, McHenry CL, Stockton DW, Rammesmayer G, Lupski JR, Antinolo G, et al. 2005.
Retinal degeneration associated with RDH12 mutations results from decreased 11-cis retinal synthesis due to disruption of the visual cycle. Hum Mol Genet 14: 3865-3875.

Tucker BA, Scheetz TE, Mullins RF, DeLuca AP, Hoffmann JM, Johnston RM, Jacobson SG, Sheffield VC, Stone EM. 2011. Exome sequencing and analysis of induced pluripotent stem cells identify the cilia-related gene male germ cell-associated kinase (MAK) as a cause of retinitis pigmentosa. Proc Natl Acad Sci 108: E569-E576.

Vithana EN, Abu-Safieh L, Allen MJ, Carey A, Papaioannou M, Chakarova C, Al-Maghtheh M, Ebenezer ND, Willis C, Moore AT, et al. 2001. A human homolog of yeast pre-mRNA splicing gene, PRP31, underlies autosoma dominant retinitis pigmentosa on chromosome 19q13.4 (RP11). Mol Cell 8: 375-381.

Woods CG, Cox J, Springell K, Hampshire DJ, Mohamed MD, McKibbin M, Stern R, Raymond FL, Sandford R, Malik Sharif S, et al. 2006. Quantification of homozygosity in consanguineous individuals with autosomal recessive disease. Am J Hum Genet 78: 889-896.

Wright AF, Chakarova CF, Abd El-Aziz MM, Bhattacharya SS. 2010. Photoreceptor degeneration: Genetic and mechanistic dissection of a complex trait. Nat Rev Genet 11: 273-284.

Wu H, Cowing JA, Michaelides M, Wilkie SE, Jeffery G, Jenkins SA, Mester V, Bird AC, Robson AG, Holder GE, et al. 2006. Mutations in the gene KCNV2 encoding a voltage-gated potassium channel subunit cause "cone dystrophy with supernormal rod electroretinogram" in humans. Am J Hum Genet 79: 574-579.

Received June 3, 2012; accepted in revised form October 4, 2012. 


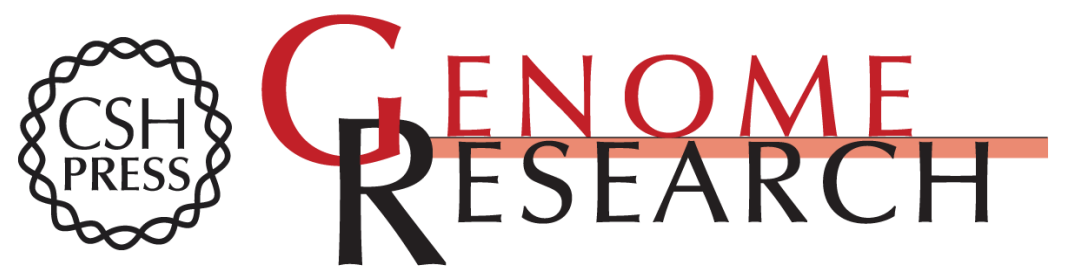

\section{Autozygome-guided exome sequencing in retinal dystrophy patients reveals pathogenetic mutations and novel candidate disease genes}

Leen Abu-Safieh, May Alrashed, Shamsa Anazi, et al.

Genome Res. 2013 23: 236-247 originally published online October 26, 2012

Access the most recent version at doi:10.1101/gr.144105.112

\section{Supplemental http://genome.cshlp.org/content/suppl/2012/11/16/gr.144105.112.DC1 \\ Material}

References This article cites 63 articles, 15 of which can be accessed free at:

http://genome.cshlp.org/content/23/2/236.full.html\#ref-list-1

Creative This article is distributed exclusively by Cold Spring Harbor Laboratory Press for the Commons

License first six months after the full-issue publication date (see

http://genome.cshlp.org/site/misc/terms.xhtml). After six months, it is available under a Creative Commons License (Attribution-NonCommercial 3.0 Unported License), as described at http://creativecommons.org/licenses/by-nc/3.0/.

Email Alerting Receive free email alerts when new articles cite this article - sign up in the box at the Service top right corner of the article or click here.

\section{Affordable, Accurate Sequencing.}

To subscribe to Genome Research go to:

https://genome.cshlp.org/subscriptions 